Meta

Journal des traducteurs

Translators' Journal

\title{
La documentation et les services linguistiques
}

\section{Nada Kerpan}

Volume 25, numéro 1, mars 1980

La documentation

URI : https://id.erudit.org/iderudit/003309ar

DOI : https://doi.org/10.7202/003309ar

Aller au sommaire du numéro

Éditeur(s)

Les Presses de l'Université de Montréal

ISSN

0026-0452 (imprimé)

1492-1421 (numérique)

Découvrir la revue

Citer cet article

Kerpan, N. (1980). La documentation et les services linguistiques. Meta, 25(1),

7-10. https://doi.org/10.7202/003309ar d'utilisation que vous pouvez consulter en ligne.

https://apropos.erudit.org/fr/usagers/politique-dutilisation/ 


\title{
La documentation et les services linguistiques
}

\author{
NADA KERPAN \\ terminologue en chef, \\ Bell Canada
}

\section{SITUA.TION ACTUELLE}

Les états de service de la documentation auprès des services linguistiques que sont la traduction, la terminologie, l'aide à la rédaction ou la rédaction même ne sont point impressionnants.

La documentation, discipline nouvelle, est essentiellement centrée sur le contenu du document - livre, périodique, article, communication, etc. - et vise à une exploitation fonctionnelle de l'information ainsi obtenue. Envisagée sous cet angle, l'opération de documentation connaît, dans les services linguistiques, un rythme lent et mal réglé. Envisagée comme activité de recherche, pratiquée par les langagiers ${ }^{1}$ que sont les traducteurs, les terminologues, les conseillers en rédaction ou les rédacteurs, elle demeure méconnue et parfois même inconnue.

A certains, elle apparaît comme une simple vogue, presque futile et, de surcroît, onéreuse en argent et en temps. A plusieurs, elle se présente comme un élément inévitable de notre société de services ou mieux du secteur quaternaire que constitue le bouillonnement actuel de l'information. Pour d'autres, enfin, elle est la condition sine qua non d'un travail de qualité en traduction, en terminologie ou en rédaction.

Aussi convient-il ici de s'expliquer pourquoi la documentation - discipline ou processus documentaire - n'est pas encore bien accueillie ou totalement admise par les services linguistiques. Il convient, en outre, de dégager le rôle qu'elle peut y jouer et, par voie de conséquence, d'indiquer la place qu'elle doit y occuper.

\section{CAUSES}

\section{Caractère général des textes}

$\mathrm{Si}$, à l'heure actuelle, la fonction de documentation n'a pas sa place aux services linguistiques ou n'y a pas suffisamment de reconnaissance, c'est qu'hier encore les besoins en justifiaient beaucoup moins la nécessité. Les traductions ne présentaient pas auparavant, de façon continue et massive, le caractère technique

1. Terme proposé par MM. Pierre Marchand et Paul Hubert de la Société des traducteurs du Québec. 
qu'elles ont maintenant. Voilà un peu plus d'une décennie, elles étaient notamment dans l'entreprise - de nature plutôt générale : textes d'information, textes administratifs, correspondance, par exemple. Le traducteur n'était donc pas systématiquement confronté à des domaines techniques, dont il lui aurait fallu appréhender les notions et rechercher la terminologie. Ainsi, le besoin de documentation était moins évident et profond. On s'y attardait à peine puisqu'il était, par ailleurs, acquis que le traducteur était un maître de la langue, que ses langues de travail ne devaient point avoir de secret pour lui et qu'il n'avait, somme toute, qu'à faire appel à ses propres ressources linguistiques, aux virtualités de la langue ainsi qu'aux ouvrages généraux de langue pour traduire les textes les plus divers. $\mathrm{Et}$ si besoin réel de documentation il $\mathrm{y}$ avait, on ne pouvait que le reléguer au second plan faute de moyens effectifs à sa disposition.

\section{Non-initiation à la documentation}

Par ailleurs, les services de traduction et de terminologie étaient le plus souvent assurés par des personnes dont les études générales ne sensibilisaient pas suffisamment à la nécessité de la documentation et d'un processus documentaire rigoureux, pourtant essentiels dans le cas de travaux spécialisés ou techniques. De plus, l'absence d'encadrement chez le nouveau venu à la traduction, l'intérêt vif ou presque exclusif pour une bonne langue générale, un certain attrait pour les créations lexicales ou les élégances stylistiques, ainsi que l'ignorance de ce qu'on nomme aujourd'hui «langues de spécialité » ne permettaient guère de découvrir la pertinence d'une fonction de documentation organisée ou d'une pratique systématique de la documentation.

\section{Insuffisances de l'enseignement}

L'enseignement même de la traduction était, il faut le reconnaître, lacunaire. Il ne comportait point de formation ni à la documentation ni au processus documentaire. Il n'offrait pas, non plus, de formation particulière en terminologie - celle-ci ne s'étant pas encore différenciée ni démarquée par rapport à la traduction. Par ailleurs, les textes d'exercice demeuraient trop souvent d'ordre général ou peu spécialisé. Enfin, les lexiques bilingues semblaient fournir suffisamment de réponses pour que l'on se permît - dans les cas où ils étaient muets sur certains termes - de produire sa propre traduction ou adaptation du terme de la langue de départ. Ce n'est que depuis une décennie que les universités ont inscrit la documentation en tant que telle à leurs programmes de traduction et que les professeurs de version ou de thème initient les élèves - avec plus ou moins d'insistance, selon les cas - à la recherche documentaire.

\section{Résistance au changement}

Dans les services linguistiques, les textes sont aujourd'hui de tous genres et de technicité diverse. La variété et les difficultés posées par l'information à transmettre requièrent donc une adéquation en moyens qui, eux, doivent assurer une rapidité d'exécution ainsi qu'une bonne qualité de traduction, de terminologie ou de rédaction. Ces moyens sont des ouvrages, des banques de documentation ou de terminologie, des spécialistes et, il va de soi, la pratique même du processus 
documentaire. Toutefois, on se heurte trop souvent encore à une indifférence sur ce point, à une paresse quand ce n'est à un refus tenace de changer des habitudes de travail, d'où - comme on le signalait précédemment - l'activité documentaire est plutôt absente. Cela revient à dire que l'on continue de mal comprendre et de mal pratiquer le processus de traduction. En effet, celui-ci ne comporte pas une simple traduction des éléments du discours, mais exige l'emploi de la terminologie et des unités fonctionnelles propres à la langue de spécialité et, fondamentalement, la compréhension du texte. On se trouve ainsi en présence de la réalité qu'est une langue de spécialité qui - comme la langue générale - ne saurait souffrir d'entorses graves à ses règles. Sans recherche documentaire, les écarts prennent ou la forme de traductions littérales qui, peu à peu, marquent et faussent la langue du domaine, ou d'erreurs de sens, qui risquent d'avoir des suites funestes.

\section{Caractère onéreux de la documentation}

Il est vrai que le coût d'acquisition d'une documentation générale ou spécialisée - complète ou fondamentale - est élevé en soi. Par ailleurs, la fonction de documentation exige beaucoup de temps. C'est, en effet, l'investissement en heures de travail réclamé par la sélection, l'acquisition, le traitement et l'exploitation qui semble prohibitif ou inusité, se traduisant le plus souvent par la création même d'un poste de documentaliste. Puis la documentation-consultation signifie - pour encore trop de chefs de service, de chefs d'équipe, de traducteurs ou pour les pseudo-terminologues - des heures de non-production; la recherche quelle qu'elle soit n'a jamais bonne presse et pourtant elle est le nerf de la production. L'urgence des travaux et le rendement à très court terme demeurent toujours les éléments prioritaires et déterminants si bien que, malgré certaines intentions fermes d'imbriquer le processus documentaire au calcul des délais, on écarte encore trop souvent l'élément pourtant fonctionnel et rentable qu'est la documentation.

\section{RÔLE DE LA DOCUMENTATION}

\section{Rôle d'information et de formation}

Face à la multitude de documents à traduire ou à rédiger et aux nombreux termes et terminologies à établir, le traducteur, le rédacteur ou le terminologue parce que peu souvent spécialistes d'un domaine ou faiblement spécialisés dans un secteur donné - ne peuvent échapper à l'obligation d'un processus documentaire continu et rigoureux et, partant, à la nécessité de disposer d'une documentation fonctionnelle. Il ne faut pas oublier, en effet, que tout autant la traduction, la rédaction que la terminologie se fondent d'abord sur l'exploration du sens ou des notions pour ensuite aboutir à la forme, qui est le texte ou le terme.

Par ailleurs, il est, en général, exigé du traducteur, du rédacteur et du terminologue une telle polyvalence que celle-ci démontre, à elle seule, le bien-fondé de l'opération de documentation. Nul esprit, il va de soi, ne peut appréhender la masse d'information actuelle, et notamment lorsqu'on doit l'interpréter ou la transposer dans une autre langue, si on n'a à sa portée les moyens et le loisir de se 
documenter. La polyvalence n'a jamais servi d'excuse et ne pourra jamais le devenir dans les cas d'erreurs de traduction ou d'infractions aux langues de spécialité.

La documentation a donc foncièrement un rôle d'information et de formation à jouer auprès du traducteur, du rédacteur et du terminologue. Elle permet de respecter cette règle fondamentale qu'est l'exactitude, la précision, la fidélité au sens. Elle permet aussi d'acquérir des notions sûres et structurées dans les domaines abordés. Le produit de traduction, de terminologie ou de rédaction qui en résultera n'en sera que meilleur et ne provoquera pas (comme il arrive trop souvent, hélas) la méfiance du donneur d'ouvrages, du spécialiste ou de l'usager du texte.

\section{Rôle linguistique}

La documentation a, en outre, un rôle purement linguistique : celui de fournir la terminologie et le style propres au domaine. C'est non seulement au terminologue qu'incombe la tâche d'exploiter la documentation sous cette forme, mais également au traducteur et au rédacteur. C'est d'ailleurs là l'autre fidélité à laquelle ceux-ci sont tenus : la fidélité à la forme.

En fait, on peut appliquer à la documentation même les trois objectifs formulés, par Robert Dubuc, pour la terminologie : réduire la marge d'intraduisibilité (ou d'incompréhension, peut-on ajouter), préserver le caractère idiomatique de la langue d'arrivée, et améliorer le rendement quantitatif et qualitatif ${ }^{2}$.

\section{PLACE DE LA DOCUMENTATION}

La documentation est un élément vital des activités langagières décrites ci-dessus; elle doit en constituer la pierre angulaire. La terminologie la proclame inhérente à son processus et «c'est parce qu'ils sont essentiellement des communicateurs que rédacteurs et traductcurs doivent se tourner vers elle ${ }^{3}$ ». Aussi faut-il lui accorder, sans tarder et dès le départ, une place privilégiée dans l'organisation et la production de services linguistiques. Elle se révèle vite d'une rentabilité mesurable, sous le rapport de la quantité et de la qualité, tant pour chacun des professionnels des services linguistiques que pour l'exploitation du service en tant que tel. La documentation, discipline ou recherche, est un investissement et un outil de saine gestion que d'autres groupes que les services linguistiques savent apprivoiser et intégrer à leur fonctionnement quotidien. «La traduction artisanale est-elle condamnée? », se demandait, en 1975, Jacques Poisson ". Oui, tout comme la terminologie et la rédaction artisanales et ce, de toute évidence, à la fin de notre $\mathrm{XX}^{2}$ siècle.

2. Robert Dubuc, «Terminologie et traduction », vire Congrès de la Fédération internationale des traducteurs, Montréal, 14-18 mai 1977.

3. Robert Dubuc, «Documentation et communication fonctionnelle», causerie prononcée

3. Robert Dubuc, "Documentation et communication
à la Société des traducteurs du Québec, mai 1978 .

4. Jacques Poisson, « La traduction artisanale est-elle condamnée? », Meta XX, no 2 , juin 1975, p. 129-140. 